\title{
Towards Service Awareness and Autonomic Features in a SIP-Enabled Network
}

\author{
Giuseppe Valetto, Laurent Walter Goix, and Guillaume Delaire \\ Telecom Italia Lab, Via Reiss Romoli 274 -10148 - Torino, Italy \\ \{giuseppe.valetto, walter.goix, \\ guillaume.delaire\}@tilab.com
}

\begin{abstract}
Next-generation communication infrastructures can become autonomic only if they can leverage some form of awareness about themselves and the services they deliver. Such awareness can be reached by disseminating across the network a proper amount of multi-faceted knowledge. We have started to identify a set of basic capabilities that provide a degree of serviceawareness and enable various autonomic behaviors, oriented towards sustaining communication services within a SIP-enabled network. We have designed network features that support those capabilities, in particular focusing on awareness features relevant to service deployment, monitoring and exposition, and we have built those features natively into the control plane, relying upon the SIP Event Framework specifications. We have also defined a set of scenarios that exploit the service awareness introduced in the network for various autonomic purposes.
\end{abstract}

\section{Introduction}

In the area of Information Technology (IT), much attention has been recently given to new ways to respond to the rapid growth in complexity and scale of today's distributed software systems and services. That trend has generated a wealth of new research, often referred to as autonomic computing [2], aiming at providing distributed software ensembles with a set of adaptive capabilities geared towards its selfconfiguration, self-healing, self-optimization and self-protection [1]. Also the field of communication technology is experiencing these days a tumultuous growth, similar to that observed in the IT field, and is thus in need of equivalent self-* provisions; this has recently led to research on autonomic communication systems.

All autonomic systems must be capable of automated and dynamic adaptation, and, to that end, one key enabling factor is awareness. Both self-awareness and environment-awareness are necessary, leading to proactive adaptation - based on evaluation of the global state of the system, as well as reactive adaptation - in response to conditions occurring within the execution environment. Next-generation networks can therefore become autonomic in a significant way only if they become aware of (i.e., can tap on and leverage) a wealth of information about themselves and the role they play in delivering communication-intensive services.

The original version of this chapter was revised: The copyright line was incorrect. This has been corrected. The Erratum to this chapter is available at DOI: 10.1007/978-3-540-32993-0_29

I. Stavrakakis and M. Smirnov (Eds.): WAC 2005, LNCS 3854, pp. 202 -213, 2006.

(C) Springer-Verlag Berlin Heidelberg 2006 
The role of knowledge and awareness is well recognized and accepted in Autonomic Communication; however, it is often summarized with the all-encompassing term of context awareness (see for example [21]), which tends to blend together a number of very different aspects. The concept of awareness is instead multi-dimensional by nature: we maintain that - while a common framework for leveraging awareness dimensions is necessary, as advocated for example in [15] - the characteristics of each of those domains should be studied separately, each on the basis of its different contribution.

We are particularly interested in service awareness, defined as the ability by the network to understand the services it provides, in terms of their nature, their state and their characteristics. That issue seems quite tightly and directly linked to enabling adaptive mechanisms that relate to the nature of the communication services carried by that network. In fact, communication infrastructures have gradually been moving away from the end-to-end, or "pipe" metaphor: increasingly - as exemplified by active networks [10] - the communication infrastructure is an actor that takes an active interest also in knowing (and, in turn, shaping) how the communication takes place and what service is carried out. However, to fully enable the idea of networks that can adjust themselves to the conditions and demands imposed upon them by services, the level of service awareness in the networks must be raised.

Service awareness is about leveraging knowledge that can be derived - in the terms used in [20] - both from the data and the control plane. We are working on introducing forms of service awareness natively within network protocols. The work we present focuses on endowing an infrastructure for advanced telecommunications services - based on the SIP signaling protocol [3] - with facilities for communicating information related to service monitoring, deployment and advertising (i.e. exposition). In this paper, we describe how we have developed and experimented with those facilities on top of SIP, the kind of information they provide, and how the level of service awareness made available enables a number of service-oriented autonomic scenarios.

\section{Background}

\subsection{SIP}

SIP (Session Initiation Protocol) [3] is an application-layer control (signaling) protocol for creating, modifying, and terminating multimedia and multiparty sessions, e.g. Internet telephone calls over an IP network.

SIP entities (typically, users or services) are accessible through a SIP address, or SIP URI, whose format is sip:username@domain.

A typical SIP network is made of proxy servers or proxies, to route requests to the user's current location, which can also authenticate and authorize users for services and implement call-routing policies. Typically, services are provided by SIP Application Servers (AS), which run software that implements the application logic, and are accessed by users through the proxies.

\subsection{The SIP Event Framework}

The SIP Event Framework [4] is a mechanism for asynchronous event notification over SIP; it comprises the following logic entities: 


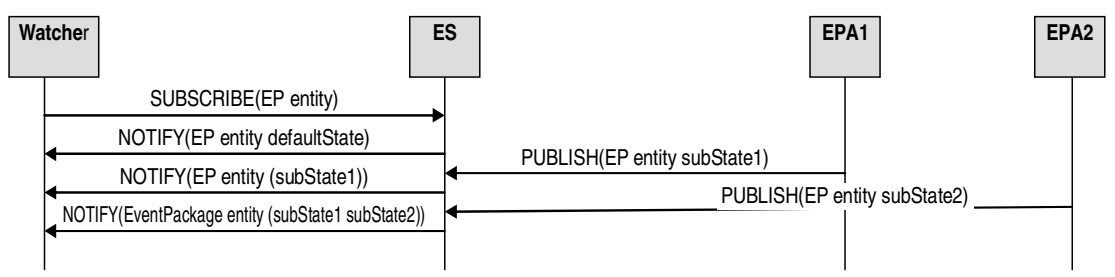

Fig. 1. Generic call flow of the SIP Event Framework

- Watcher: an entity interested to receive information: it declares its interest via an explicit subscription created with a SIP SUBSCRIBE message.

- Event Server (ES): notifies Watchers with the requested information, through a SIP NOTIFY message. How the ES produces or retrieves the information to be notified is out of the scope of the framework itself.

- Event Publication Agent (EPA) - or Event User Agent (EUA): provides the ES with information through the SIP PUBLISH [5] method. Multiple EPAs can send PUBLISH requests to the ES on behalf of the same "entity" and with respect to the same subscription. In that situation, the ES must aggregate the information prior to notify Watchers.

The above-described mechanism is general enough to allow a variety of notification services, named "Event Packages" (EPs). A generic event notification scenario using the SIP Event Framework is described in Fig. 1.

The ES is the central point of the SIP Event Framework, which gathers and propagates information from and to SIP entities, carrying out the manipulation of data between the information published by the EP and the information notified to watchers. Such manipulation includes for example data filtering, aggregation or transformation. It is also in charge of protecting sensitive information, by managing subscription access based on the identity of the watcher and its related privileges.

Each EP defines its own XML format to describe specific events. It also defines any composition and transformation rules that must be applied by the ES to aggregate information PUBLISHed from several EPAs, before proceeding to NOTIFY them in a single event carrying an aggregated XML document. That aggregation feature is a peculiarity of the SIP Event Framework with respect to most event-based communication buses. Before sending this aggregated document to the Watchers, the ES can further apply filters defined by local policy or by Watchers when subscribing.

\section{Introducing Service Awareness in a SIP Network}

We have developed some service awareness capabilities in a SIP-enabled network, as a set of Event Packages, for the exchange of non-functional information related to the state of services as well as network elements. Our interest in SIP comes from the possibility to enable an existing control layer that is being currently deployed in advanced service-based networks, to evolve gracefully to include autonomic behaviors, without the introduction of ad-hoc additional protocols. That provides a 
good framework for practical experimentation and evaluation of service awareness features in next-generation networks.

Our work also allows encompassing SIP-based terminals in a service-aware communication environment, which is advantageous in a telecom context, given the large number and wide variety of terminals operating on the service network.

By choosing SIP, we have also been able to leverage the characteristics of its wellspecified Event Framework, which seems particularly indicated for the native integration of situation (and - specifically- service) awareness within the control plane of Next Generation Networks. The relative simplicity of defining Event Packages for the SIP Event Framework supports the process of extending the awareness information with additional dimensions. It thus permits to promptly provide more sophisticated levels of service awareness, which could be used for autonomic concerns ${ }^{1}$.

For starters, we have focused on Event Packages dealing with service monitoring, deployment and advertising, whose semantics enable to communicate about and to control what happens during the post-development lifecycle of a service, starting from the moment in which it is ready to be rolled out on the network. Hereby we motivate our choice, highlighting the importance of monitoring, deployment and advertising information for autonomic scenarios.

The monitoring Event Package provides a generic capability to collect information about and inspect the state of entities within the network, as they evolve over time. That is a basic feature for any autonomic system, since it enables the necessary levels of self-awareness needed for introspection and diagnosis. The monitoring package, as we designed it, supports the collection of different kinds of data, and can be tailored towards different network elements and services. The subscription mechanism, furthermore, allows filtering of events in various ways, and the construction of multiple monitoring views that respond to different reporting needs and can be directed to diverse recipients.

While monitoring constitutes on its own only a pre-requisite for the development of autonomic behavior, the deployment Event Package addresses directly some concerns related to the four major self-* autonomic areas. Deployment events provide notification means and commands for the installation, configuration, activation, deactivation and retirement of service and service components on SIP Application Servers, as well as the arming and disarming of SIP proxy triggers used to route requests to those services. As such, deployment events provide a set of primitives that can be leveraged, in the first place, for the automated and controlled (re-) configuration of services on top of the nodes of a SIP network. In particular, a combination of deployment actions enable the configuration of arbitrarily complex composed services, by moving, adding or substituting components on a topology of available network elements. Deployment primitives remain coarse-grained and as such do not cover the whole (re-)configuration spectrum. However, especially when used in conjunction with the monitoring Event Package, they can also be effective

\footnotetext{
${ }^{1}$ Notice how SIP already includes protocol-level means to provide a level of person awareness to the network, called SIP Presence [6]. Presence information in a SIP network is aimed at representing the state of users and their terminals and devices; the basic presence information can also be extended to capture more details, as required by applications.
} 
towards other autonomic concerns: for example, self-healing and self-protection- as exemplified by the Willow survivability architecture [17], which leverages the Software Dock deployment engine [18]; or self-optimization, for example to increase the instances of critical services or components present on the network, in the face of bottlenecks and request surges, or to modify the routing scheme, in order to streamline communications.

Finally, the advertising Event Package deals with service exposition, i.e., provides interested watchers, such as (but not limited to) user agents and terminals, with notifications on available services and their characteristics. The advertising Event Package is used to communicate information such as the access modality and channel to the service, and the entry point to the service network (proxy). Advertising events, therefore, are instrumental to push service awareness towards the edge of the network. Service exposition, as we implemented it through the advertising Event package, can be tightly coupled with deployment; that helps in making new services immediately and automatically available / unavailable to all user agents (or to selected subsets) as they are successfully activated / deactivated, as a logical extension of configuration or healing scenarios. Such feature may also affect optimization, as seen from the network edge, for example for dynamically planning and dimensioning the ratio between user agents and proxies. Finally, advertising can also be used within the network itself to adapt on the fly the view of available services also to other services, which has clear beneficial implications for autonomic communication scenarios that intend to deal with spontaneous service aggregation and composition.

The rest of this Section describes in detail the Event Packages introduced above.

\subsection{Monitoring}

The goal of the monitoring Event Package is to provide information about the state of a network element or a service over time to interested monitoring parties, or watchers. Network elements typically publish information about their own state, and separate information on behalf of each service (or trigger, in case of a proxy) they host.

In the simplest case, the monitoring information consists in on/off availability data. To detect the unavailability of network or service entities, keep-alive mechanisms are used, requiring monitored elements to frequently refresh their availability information. That makes available a "heartbeat" that enables to notify watchers in near realtime whenever a refresh event is not received in time.

Enrichment of the basic heartbeat data is made possible by the extensibility of the XML schema for monitoring events. Our architecture envisions that a single network element may incorporate many monitoring components, each of them publishing different information, using different XML namespaces. The notification of arbitrarily rich monitoring data produced within a service component and issued by the hosting node is therefore easily enabled, and entirely application-dependent.

On the other side, watchers can subscribe to raw data or - taking advantage of the filtering and data composition mechanisms provided by the Event Framework - to some aggregate data, based on several criteria, such as network element identifier, or type (e.g. AS or proxy), service identifier, etc. Some examples of subscriptions with different purposes are given below: 
- Auto-discovery: all known information about all network elements

- Network Element monitoring: all known information about a single network element, such as an AS, including the state of the node and all its hosted services

- Service monitoring: all known information about a service, across all network elements that host it

- Load distribution: load information compiled for all elements of a certain type (AS, proxy, service)

\subsection{Deployment}

The deployment Event Package is substantially more complex that the monitoring one, since its goal provide a complete view of what happens on a SIP network during the service deployment, retirement and upgrade process.

Deployment events circulate in the network on the basis of an XML deployment document. That document is intended to prescribe a workflow of deployment steps (or actions). When the document is created, a corresponding event is routed to network elements involved in some step of the workflow, i.e., the deployment targets. Each target checks whether the action(s) prescribed for that target can be performed immediately, or depends on actions that must yet take place upon other targets.

In the former case, the target sends feedback through a PUBLISH request, indicating that it has finished processing the action, to indicate either success or failure. In the latter case, the network element will perform its action only when it receives the notifications indicating that those other actions are completed.

This way, once the process is under way, notification events that are issued and propagated from network elements involved in the workflow collectively enable to maintain a coherent state trace of the deployment procedure across all targets.

XML extensibility is also leveraged in the Deployment EP, for instance to provide network elements with data relevant to the deployment of specific services, such as their initial configuration, etc.

At startup, we envision that a network element subscribes to all deployment processes. If it is an intended target in some already running deployment processes, the Deployment Server returns all relevant events aggregated in the body of a NOTIFY. This mechanism enables network elements to remain up to date about deployment processes that may have been triggered on the network at any time in which they were not connected, which is helpful for automated recovery and leads to self-stabilization.

Fig. 2. exemplifies a typical automated deployment scenario, in which a new service must installed and activated. The whole deployment process is activated through a single PUBLISH request from some deployer entity, which may initiate the process autonomously, or due to an external stimulus, such as an event containing monitoring information. This scenario envisions four automated steps: (1) a service is deployed on a SIP AS, (2) a corresponding trigger is installed on a SIP proxy at the same time, (3) once the trigger is installed, the service may be invoked, so the service is activated on the AS, (4) once the service is active, the proxy can activate its trigger to route incoming requests to the AS. In this scenario, the deployer entity in the network subscribes to the deployment process itself, in order to watch over its progress. 


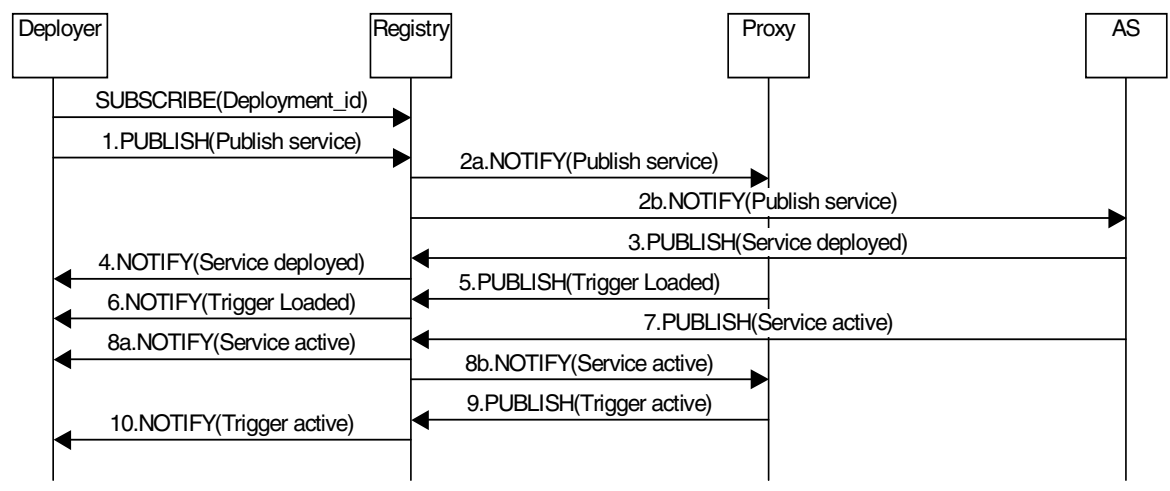

Fig. 2. A deployment scenario - installing and activating a service

\subsection{Advertising}

The advertising Event Package communicates rich information about available services: we have defined it on the basis of the Presence Event Package [6]. Our package defines an extension to the Presence XML schema [7], which enables to notify a watcher (e.g. a user device) with service availability, how and where to access the service, and optionally any additional information useful for service invocation. It has been designed also to allow subscribing to list of services, according to categorization mechanisms that can be user-, application- and domain-specific. In some cases, the number of services within each list may vary over time.

Typically, when starting up, user terminals subscribe to one or more list of services using the standard presence subscription mechanism defined in [6], and get notified about services available on the SIP network, together with the corresponding additional advertising information. Whenever a new service is available, or becomes unavailable, a PUBLISH request containing new service information is issued to the advertising ES, which triggers notification of this change to all interested watchers. For scalability reasons, a hierarchical architecture has been designed that geographically distributes terminal subscriptions over outbound advertising ES, which in turn subscribe to service lists on a central master server.

Notice that, advantageously in an autonomic scenario, advertising can be linked to monitoring or deployment information, to dynamically reconfigure user terminals in response to new services as well as changes in availability of existing services.

\section{Building Autonomic Scenarios Using Service Awareness}

In this Section, we outline how some typical autonomic scenarios that are often used to exemplify common self-* concerns are enabled in a SIP network by the service awareness features we have introduced, in particular using the above-defined Event Packages. Our purpose is to remark how even relatively straightforward forms of service awareness provide a sufficient level of support to a variety of basic autonomic applications. 


\subsection{Self-configuration}

The first, obvious configuration scenario regards the adaptive deployment of a service on a certain portion of the network, that is, on a certain number of AS and proxy elements, each of which must be individually equipped with the appropriate triggers and software features. The foundation of this scenario is of course the automated deployment facilities provided by our deployment Event Package.

Automated deployment immediately becomes adaptive when some controlling entity, e.g., the original deployer entity, as seen in Fig. 2, can modify on the fly our deployment document, and issue it as a subsequent event that updates the deployment workflow. That controlling entity can act for various reasons: on the basis of a particular state reached in the workflow, or in response to external stimuli (e.g. incoming monitoring information), or to modify proactively the deployment process, based on some policy decision. Notice how the SIP Event Framework naturally supports adaptive deployment, through its keep-alive, aggregation and trasformation features.

Another configuration scenario regards the self-adaptive static load balancing of a service $^{2}$, which relies principally on monitoring events. In this scenario, a highthroughput proxy acts as a load balancer to a cluster of AS nodes, all providing the same service. The proxy can subscribe to monitoring events published by the AS in the cluster and the running service instances hosted by that cluster. That way, it is made continuously aware of the runtime properties of services (number of requests being processed, number of error generated, etc.), as well as the state of the elements in the cluster (CPU load, network load, length of request queue, etc.). Further monitoring information, expressed in whatever measurement unit is appropriate for the service at hand, can also be considered. The proxy has installed specialized logic and algorithms, which, by subscribing to and processing that monitoring information, decides on the fly how to distribute incoming requests. Additionally, another monitoring entity can be added to this scheme, in charge to decide when to equip the proxy with a new logic, in order to modify on the fly the distribution profile it uses, and optimize it with respect to higher-degree circumstances.

\subsection{Self-healing}

The combination of monitoring and deployment events can address various fault recovery scenarios. Monitoring events can be issued by AS elements to signal different kinds of application-level faults, such as failures of the deployed service software. Also network-level faults can be similarly signaled by the affected AS and proxy elements. A deployer entity with specialized logic on board that subscribes to those events can react by issuing appropriate re-deployment events as a countermeasure. For application-level faults, such as software crashes, it can decide to re-activate the crashed component on the same AS. For network-level faults, it can re-configure the service by deploying onto different network elements new instances of the unreachable service, and/or change the routing of proxies, to overcome network partitioning.

${ }^{2}$ Static load balancing, as opposed to dynamic load balancing (described in Sec 4.3.), involves the over-provisioning of the service, with enough active instances activated on a number of AS nodes, to ensure they can collectively handle any reasonably foreseeable request load. 
The same approach can also be used for fault avoidance, assuming sufficiently rich monitoring information, coupled with sophisticated analysis and proactive logic on board of the deployment service acting as a subscriber. In that case, the accumulated information about the service state can reveal conditions that might lead to a fault, and the proactive interventions can consist of an appropriate set of deployment events. For instance, in case the memory occupation by some entity can be monitored, whenever a certain threshold is passed a fault avoidance strategy might be to de-activate and then shut down the offending entity before it crashes hard - possibly taking down a number of important user sessions - and start a fresh copy anew. Notice that this scenario can be made increasingly sophisticated by embracing the idea of controlled, hierarchical reboots, like in Recursive Restartability approaches [19]: if we envision a service as a collection of features running on various AS instances, it may be possible to shutdown and restart a single feature, or a single AS, or subsets of features and AS, in order to get rid of the problem at the finest possible granularity level, without perturbing service components that remain in good shape.

\subsection{Self-optimization}

An optimization scenario is that of dynamic load balancing. On the basis of load monitoring events originating from the AS elements and their resident services, a subscribing deployer entity can react to a request peak for a certain service by scaling up, that is, deploying on the fly one or more additional instances of that service onto AS elements that happen to be able to handle additional computational and communication loads, and re-configuring the service trigger of the outbound proxy for that service accordingly. Conversely, when the peak is over, the deployer entity can decide to scale down, deactivating or retiring some of the newly deployed service instances, thus releasing some resources. Dynamic load balancing reduces the need for overprovisioning; thus, it enables optimized usage of the resources of the network, without compromising availability and responsiveness.

Another optimization scenario, at the edge of the network, involves advertising in combination with monitoring. It aims at resolving cases in which a proxy is overloaded by the traffic generated by its assigned user agents: to optimize its ability to efficiently route requests, that situation can be monitored, and advertising events can be issued to re-assign some of the user agents involved to less busy proxies.

\subsection{Self-protection}

Many protection scenarios, which aim at easing the effects of or altogether thwarting attacks to the service network, can be approached in ways that are analogous to those used for self-healing. From the point of view of autonomic communication, the concepts of fault recovery and fault avoidance apply also to self-protection, with the "semantic" difference that the fault in this case is not fortuitous, but maliciously inoculated into the network. Another difference is that faults caused by attacks tend not to remain local, but to actively spread across the network.

Deployment features can be helpful to counter that to a degree, in order to willingly cause the partitioning of the service network, and "quarantine" the portion under attack, thus limiting the effect of that attack. Changing the deployed triggers on 
SIP proxies is a very effective way to cause transient network partitioning as needed. To achieve self-protection via partitioning, however, it is necessary to put in place rather sophisticated and efficient (hence resource-intensive) analysis facilities of the monitoring information that circulates in the network, to quickly and proactively recognize anomalous patterns and behaviors that reveal an attack before it spreads too widely. How to deal with that challenge is a large part of the research on intrusion detection, which is itself based on awareness about the behavior of entities residing on the network, and the recognition of "suspicious" or non-motivated variations of that behavior. We are currently investigating how to inject that kind of awareness specifically in a SIP network, in particular as an evolution of our monitoring facilities.

\section{Related Work}

Several awareness domains are actively being researched. For example, personawareness in networks (e.g. with PANs), as well as services, increasingly tries to tie the individual world of users to their usage of communication, by taking into account intrinsic and explicit personal demands (see for example I-centric communications [13]). Another example is space-awareness which is obviously very important in contexts like mobile communications and sensor networks, but more subtly also for situating an ensemble of communication entities within a generic multi-dimensional metric space, which can be used to define and measure inter-relationships among those entities (as proposed in spatial computing [14]). Many other dimensions are sometimes collectively encompassed by the generic term of context awareness, and variously exploited, for example in ad-hoc networking (as in [16]). In general, however the systematic investigation of each single awareness dimension, and its relevance for enabling autonomic communication, remains an open research endeavor. In the extreme, that investigation can lead to the introduction of a full-fledged knowledge plane [15] in between the data transport plane and the application plane.

In the context of SIP networks, the Event Framework is receiving significant interest especially as a means for adapting services on the basis of users' context (user awareness). Works like [8] and [9] follow that approach, in particular for the adaptive configuration of User Agents, which we try to support in a general way with our service advertising Event Package. We are not aware, however, of other works besides ours - which use the SIP Event Framework to embed deployment and monitoring primitives in the network, in order to support adaptation of services.

Similar capabilities are instead found in the field of active networks. NESTOR [11], for example, with the complement of the JSpoon language [12], makes service components aware of and accessible by a self-configuration management layer, and can achieve reactive autonomic behavior similar to that enabled by our monitoring and deployment Event Packages.

\section{Conclusions and Future Work}

Our work aims at experimenting with service awareness capabilities, as enablers for a variety of autonomic behaviors within a SIP-enabled network. The originality of our 
contribution is twofold: first of all, since we have chosen to implement those capabilities as Event Packages on top of the SIP Event Framework, they have become native to the SIP control network; second, they are modular and can be easily extended to cover other awareness aspects. Together, those two characteristics provide the foundations of an awareness framework native to SIP, which we are incrementally designing and building within the Telecom Italia SIP-based service control platform. We are currently investigating the usage and extension of that service awareness framework, in particular along the following directions:

- Application of monitoring / deployment at the network edge, i.e., on user agents.

- Extension of deployment events to provide a highly flexible sensor configuration. That would allow new sensors to be deployed and configured on network elements as needed, to dynamically enhance reporting through the monitoring feature. For example, preliminary monitoring events could be used to trigger a deployment of new specialized sensors for a given situation. We are examining dynamic Aspect-Oriented Programming (AOP) techniques to that end.

- Application of policy-based mechanisms, as a natural complement to the service awareness events that circulate in our Event Bus. Policies can be used for a highlevel, easily re-configurable implementation of the adaptation logic that is present in the autonomic scenarios proposed in Sec. 4, as well as future scenarios. Policies could be dynamically installed via deployment on watchers for specific EPs.

- Extension of advertising to support spontaneous aggregation behavior. Advertising can disseminate information that is needed by service components to find, negotiate with and interact with other components, for dynamic selfcomposition.

\section{References}

1. A.G. Ganek, T.A. Corbi, The Dawning of the Autonomic Computing Era, IBM Systems Journal, 42(1): 5-18, January-March 2003.

2. P. Horn, Autonomic Computing: IBM's Perspective on The State of Information Technology, at Agenda 2001 conference, Scottsdale, Az., USA, October 15, 2001, http://www.research.ibm.com/autonomic/manifesto/agenda2001_p1.html

3. Rosenberg et al., SIP: Session Initiation Protocol, RFC3261, June 2002 http://www.ietf. org/rfc/rfc3261.txt

4. B. Roach, Session Initiation Protocol (SIP)-Specific Event Notification, RFC3265, June 2002 http://www.ietf.org/rfc/rfc3265.txt

5. Niemi, Session Initiation Protocol (SIP) Extension for Event State Publication, RFC3903, October 2004 http://www.ietf.org/rfc/rfc3903.txt

6. J. Rosenberg, A Presence Event Package for the Session Initiation Protocol (SIP), RFC3856, August 2004, http://www.ietf.org/rfc/rfc3856.txt

7. Sugano, et al., Presence Information Data Format (PIDF), RFC3863, August 2004, http://www.ietf.org/rfc/rfc3863.txt

8. L. Wei, A Service Oriented SIP Infrastructure for Adaptive and Context-Aware Wireless Services, in Proceedings of the 2nd Intl. Conference on Mobile and Ubiquitous Multimedia, Norrköping, Sweden, December 10-12, 2003. 
9. D. Pavel, and D. Trossen, Context Provisioning and SIP Events, in Proceedings of the MobySys 2004 Workshop on Context Awareness, Boaston. Ma., USA, June 6, 2004.

10. D.L Tennenhouse, J.M. Smith, W.D. Sincoskie, D.J. Wetherall, G.J. Minden, A survey of active network research, IEEE Communications Magazine, 35(1): 80-86, 1997.

11. A.V. Konstantinou, Y. Yemini, and D. Florissi, Towards Self-Configuring Networks, in Proceedings of the DARPA Active Networks Conference and Exposition, San Francisco, Ca., USA, May 2002.

12. A.V. Konstantinou, and Y. Yemini, Programming Systems for Autonomy, in Proceedings of the IEEE Autonomic Computing Workshop, Active Middleware Services (AMS 2003), Seattle, Wa., USA, June 2003.

13. S. Arbanowski, P. Ballon, K. David, O. Droegerhorn, H. Eertink, W. Kellerer, H. van Kranenburg, K. Raatikainen, and R. Poplescu-Zeletin, I-centric Communications: Personalization, Ambient Awareness, and Adaptability for Future Mobile Services, IEEE Communication Magazine, 42(9), September 2004.

14. F. Zambonelli, and M. Mamei: Spatial Computing: an Emerging Paradigm for Autonomic Communication and Computing, in Proceedings of the $1^{\text {st }}$ IFIP WG6.6 International Workshop on Autonomic Communication (WAC 2004), Berlin, Germany, October 2004.

15. D.D. Clark, C. Partridge, J.C. Ramming, J.T. Wroclawski: A Knowledge Plane for the Internet, in Proceedings of ACM SIGCOMM 2003, Karlshrue, Germany, August 2003.

16. R. Gold, C. Mascolo: Use of Context-Awareness in Mobile Peer-to-Peer Networks, in Proceedings of the 8th IEEE Workshop on Future Trends of Distributed Computing Systems (FTDCS'01), Bologna, Italy, October 31-November 2, 2001.

17. J.Knight, D. Heimbigner, A. Wolf, A. Carzaniga, J. Hill, and P. Devanbum: The Willow Survivability Architecture, in Proceedings of the 4th Information Survivability Workshop (ISW-2001), Vancouver, B.C.,18-20 March 2002.

18. R.S. Hall, D. Heimbigner, and A.L. Wolf, A Cooperative Approach to Support Software Deployment Using the Software Dock, in 21st International Conference on Software Engineering, May 1999.

19. G. Candea, and A. Fox: Recursive Restartability: Turning the Reboot Sledgehammer into a Scalpel, in Proceedings of the 8th Workshop on Hot Topics in Operating Systems, Schloss Elmau, Germany, May 2001.

20. R. Sterrit, M. Mulvenna, and A. Lawrynowicz: A Role for Contextualized Knowledge in Autonomic Commnication, in Proceedings of the $1^{\text {st }}$ IFIP WG6.6 International Workshop on Autonomic Communication (WAC 2004), Berlin, Germany, October 2004.

21. A.K. Dey: Understanding and Using Context, Personal and Ubiquitous Computing Journal, 5(1):4-7, 2001. 